\title{
Routine Replacement of Tunneled, Cuffed, Hemodialysis Catheters Eliminates Paraspinal/Vertebral Infections in Patients with Catheter-Associated Bacteremia
}

\author{
Marie Philipneri Ziyad Al Aly Kamal Amin Mary E. Gellens Bahar Bastani \\ Division of Nephrology, Saint Louis University Health Sciences Center, Saint Louis, Mo., USA
}

\section{Key Words}

Hemodialysis · Catheter - Bacteremia - Epidural abscess · Osteomyelitis $\cdot$ End-stage renal disease

\begin{abstract}
Background: Management of tunneled, cuffed, central venous catheters in hemodialysis (HD) patients with bacteremia remains a challenge. Attempts to salvage the catheter with systemic antibiotics alone have been associated with increased risk of metastatic infectious complications. Methods: Retrospective case series of patients with infectious complications in a chronic dialysis unit, affiliated with a tertiary care university hospital. Results: Between 1996 and 1999, when we treated HD catheter-associated bacteremia with systemic antibiotics alone, we encountered a clustering of 8 cases of paraspinal/vertebral infections in a population of 162 patients. After changing our protocol, i.e., routine catheter exchange over a guide wire at $\sim 48 \mathrm{~h}$, while on systemic antibiotic therapy, we did not encounter any new cases of paraspinal/vertebral infections over a 15-month period. Conclusion: Our experience suggests that routine exchange of tunneled, cuffed catheters over a guide wire in HD patients presenting with bacteremia may significantly reduce serious infectious complications, e.g., epidural abscess/vertebral osteomyelitis.
\end{abstract}

Copyright $(2003$ S. Karger AG, Basel

\section{Introduction}

The maintenance of vascular access is a major problem in the management of patients on chronic hemodialysis (HD). Permanent or semi-permanent catheters for vascular access are utilized in 10-30\% of patients with endstage renal disease requiring HD [1]. Well-recognized catheter-related complications include bacteremia, exitsite or tunnel infections, and frequent clotting episodes requiring intervention. Infections account for up to $40 \%$ of these complications [2].

Management of infected HD catheters is difficult and there are few evidence-based guidelines in that regard. Several studies have shown successful treatment of catheter-associated infection without catheter removal [3-5]. However, persistent bacteremia and metastatic complications such as epidural abscess, osteomyelitis, discitis and endocarditis remain a major problem in these patients [6, 7]. Current DOQI guidelines (guideline 26) recommend treatment with parenteral antibiotics and removal of the catheter only if the patient remains symptomatic [8]. Although one report has advocated routine catheter exchange over a guide wire at 24-48 h [9], it has not been adopted as standard of care by most dialysis units, and currently there is no consensus among the nephrologists on the proper management of catheter-associated bacteremias. A 2- to 3-week course of appropriate systemic antibiotic therapy, without catheter removal, has resulted in

\begin{tabular}{ll}
\hline KARGER & $\oplus 2003$ S. Karger AG, Basel \\
$0250-8095 / 03 / 0234-0202 \$ 19.50 / 0$ \\
$\begin{array}{l}\text { Fax +4161306 12 34 } \\
\text { E-Mail karger@karger.ch } \\
\text { www.karger.com }\end{array}$ & $\begin{array}{l}\text { Accessible online at: } \\
\text { www.karger.com/ajn }\end{array}$
\end{tabular}

Marie Philipneri, MD

Division of Nephrology

Saint Louis University Health Sciences CenterSt. Louis, MO 63110 (USA)

Tel. +1 314577 8765, Fax +1 314771 0784, E-Mail philipmd@slu.edu 
catheter salvage in only $20-30 \%$ of these patients $[4,5]$. This may be due to the fact that systemically administered antibiotics have insufficient penetration into the lumen of the HD catheter [10], and thus may not kill bacteria in the biofilm within the catheter lumen [11, 12], hence the rationale for routine exchange of the infected catheter over a guide wire [9] or the use of the 'antibiotic-lock technique' $[13,14]$.

Paraspinal/vertebral infections are relatively rare but catastrophic complications of dialysis catheter infection that require surgical treatment and often leave the affected patients paraplegic or dead. These complications often follow a bacteremic episode and are associated with extended, repeated attempts at salvage of the infected catheters $[6,15]$. The time between diagnosis of bacteremia and paraspinal/vertebral infection remains uncertain.

Between 1996 and 1999 we encountered a clustering of 8 cases of paraspinal/vertebral infections among our chronic HD patients. Seven of these patients had documented bacteremia that preceded the diagnosis of paraspinal/vertebral infection by $1-20$ weeks. Based on our past protocol, the catheter-associated bacteremias were managed with appropriate systemic antimicrobial agents and the catheter was not removed in patients who had good clinical and bacteriologic response. However, after encountering the cluster of paraspinal/vertebral infections we changed our practice of attempted catheter salvage with systemic antibiotics alone, i.e., we routinely exchanged the tunneled catheters over a guide wire at $\sim 48 \mathrm{~h}$, in addition to parenteral antibiotics. When there was evidence of tunnel infection, a new tunnel was created and the replacement catheter was placed at the new site. In retrospect, we did not encounter any new cases of paraspinal/vertebral infections over the subsequent 15 months in which we adopted the new protocol.

\section{Patients and Method}

Between 1996 and 1999, we encountered 8 cases of paraspinal/ vertebral infections in a population of 162 patients on maintenance $\mathrm{HD}$ at the Saint Louis University outpatient chronic dialysis center. Chronic, dual-lumen, tunneled, cuffed catheters were used in 18-23\% of our patients during that time period. Inpatient and outpatient records were reviewed to retrieve demographic data and information pertaining to the clinical presentation, diagnosis, management and outcome. The type of HD access, interval between insertion of HD access and documentation of bacteremia, and subsequent development of paraspinal/vertebral infection were obtained. Data were collected from both hospital and dialysis unit records. The demographics of our base HD patient population were as follows: males $63 \%$; diabetes 41.5\%; African-American 81\%; Caucasian 17\%, and other races $2 \%$.
New Catheter Exchange Protocol

In hemodynamically unstable patients the catheter was discontinued immediately and HD was provided via intermittent temporary catheters until their blood cultures were consistently sterile. If the patient was clinically stable, the cuffed catheter was exchanged over a guide wire within $\sim 48 \mathrm{~h}$ of presentation with bacteremia, provided there was no evidence of tunnel infection. If there was exit site infection, or erythema or tenderness over the subcutaneous tunnel suggestive of tunnel infection, a new tunnel was created and the replacement catheter was placed at the new site.

In a retrospective review of our experience, after changing our clinical practice we found no new cases of paraspinal/vertebral infections over a 15 -month period.

\section{Technique of Catheter Replacement}

Under ultrasound guidance the vein was punctured and a guide wire was introduced into the central vein. The puncture site was extended into an about $1-\mathrm{cm}$ skin incision and the subcutaneous tissue was infiltrated with $1 \%$ Lidocaine solution to an exit site in the chest or the thigh area, depending on the site of insertion. The exit site was created with a scalpel and the cuffed catheter was pulled through the subcutaneous tunnel from the skin incision. The venous puncture site was extended and dilated up to 16 french over a guide wire. A 16-french peel-away sheath was introduced into the vena cava and the catheter was inserted through the peel-away sheath. The optimal catheter position and free flow aspiration and injection in both ports were confirmed under fluoroscopy. The catheter ports were filled with 1:1,000 dilution heparin solution. A 3.0 nylon suture was used for skin closure and to secure the catheter.

If the catheter was exchanged over a guide wire, a skin incision was made close to the venous entry point of the catheter. After exposing the catheter it was pulled back a few centimeters and divided. Hemostasis was established by clamping the proximal portion of the catheter in the vein. A guide wire was passed through the old catheter and the catheter was pulled out while direct pressure was applied over the vein. Using the same tunnel a new catheter was pulled through the tunnel into the vein through the peel-away sheath, as described above.

\section{Results}

The clinical features of the 8 patients with paraspinal/ vertebral infections were as follows: mean $( \pm$ SD) age at presentation was $62.5 \pm 10.5$ years; 7 patients were male; 5 had diabetes mellitus; 6 patients used a double-lumen, tunneled, cuffed catheter for HD, and 2 patients received HD treatments via synthetic arteriovenous (AV) grafts.

The presentation of paraspinal/vertebral infection in all cases was back pain localized to cervical (1 case) and/or lumbar region (8 cases). Two patients presented with neurological deficits suggestive of spinal cord compression. Six patients had fever at presentation. Leukocytosis (WBC $\geq 10,000 / \mathrm{mm}^{3}$ ) was present in 5 patients.

The time course of bacteremia, antibiotic administration, removal of the vascular access, and the diagnosis of 


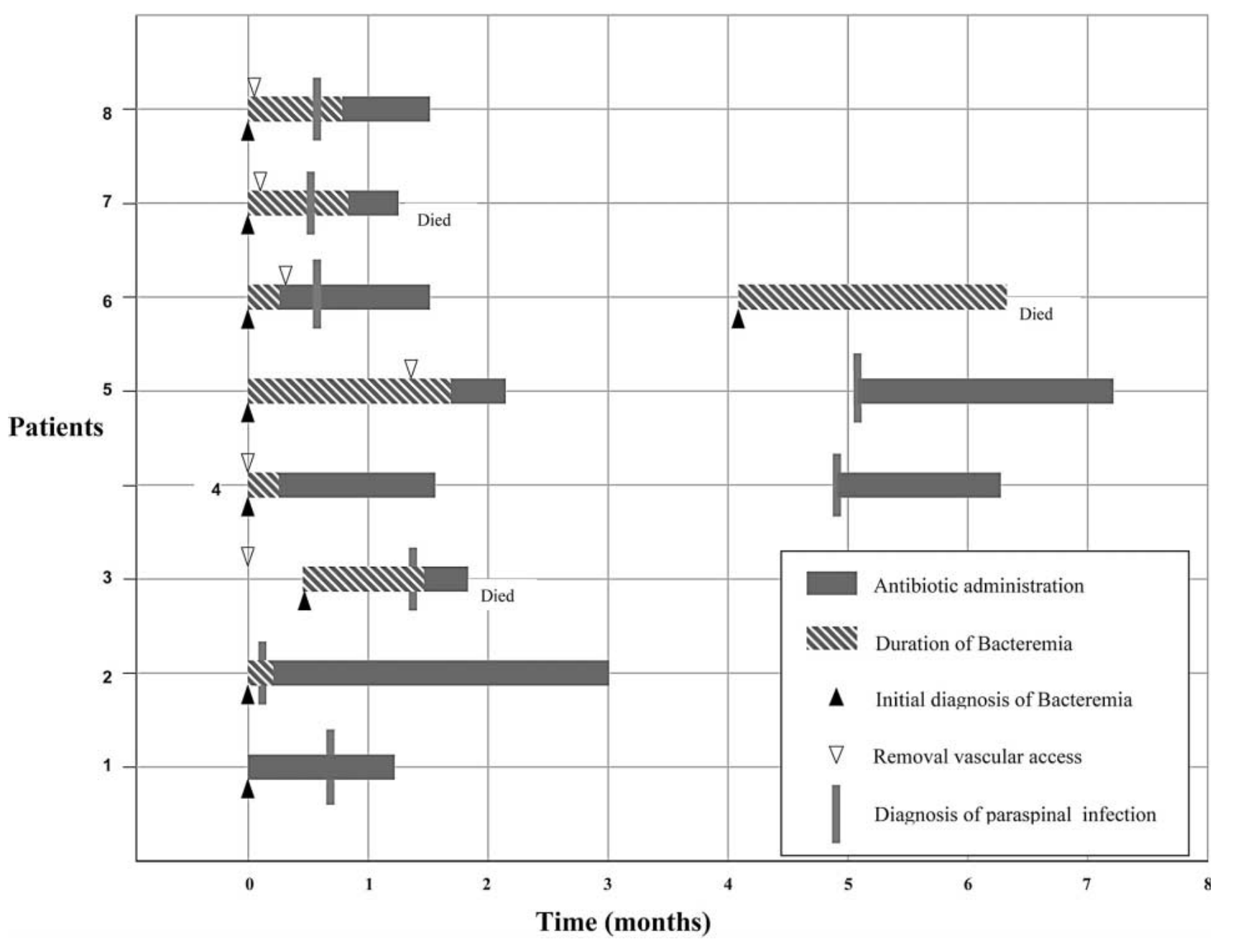

Fig. 1. The time course of bacteremia and the initial diagnosis of paraspinal/vertebral infections in the 8 patients. The timing of antibiotic administration and removal of vascular access are also provided where applicable. Patients 1 and 2 received $\mathrm{HD}$ via synthetic $\mathrm{AV}$ grafts.

paraspinal/vertebral infections are shown in figure 1. All patients had documented bacteremia that preceded the diagnosis of paraspinal/vertebral infection by 1-20 weeks. Immediately prior to this, 4 patients had sterile blood cultures diagnosed.

The microorganisms identified were vancomycin-resistant enterococcus $(\mathrm{n}=1)$ and Staphylococcus aureus species $(n=7)$ - coagulase-negative $(n=4)$, methicillinsensitive $(n=2)$, and methicillin-resistant $(n=1)$.

The diagnosis of paraspinal/vertebral infections was made by magnetic resonance imaging (MRI) in all of the 8 patients. The infections included epidural abscess, verte- bral osteomyelitis and discitis. Needle aspiration biopsy was performed in 6 of the 8 patients to confirm diagnosis. Figures 2-4 illustrate some representative MRI findings in 3 patients.

Therapy was instituted with intravenous antibiotics according to the bacteriologic sensitivities and was continued for 4-6 weeks in 7 patients, and for 12 weeks in 1 patient. Surgical debridement was performed in 4 patients. Therapy was consideredsuccessful by sterilization of the blood and the absence of recurrent bacteremia.

Over the subsequent 15-month period in which we changed our protocol, i.e., replacing the catheters at 
$\sim 48 \mathrm{~h}$ in addition to the systemic antimicrobial therapy, we encountered no new cases of paraspinal/vertebral infections in our HD patients with tunneled, cuffed catheters.

During both time periods when HD catheter-associated bacteremia was suspected based on clinical signs and symptoms of fever and chills, blood cultures and sensitivities were sent, and the patients were empirically treated with vancomycin and gentomycin until blood culture results became available, at which time, the choice of antibiotic was tailored according to the antimicrobial sensitivity of the microorganism identified.

Fig. 2. MRI of the lumbosacral spine with gadolinium contrast. An anterior epidural abscess (arrow) is seen at the L3-L5 level, significantly effacing the cal sac. Biopsy revealed acute inflammation, and culture grew vancomycin-resistant Enterococcus faecium.

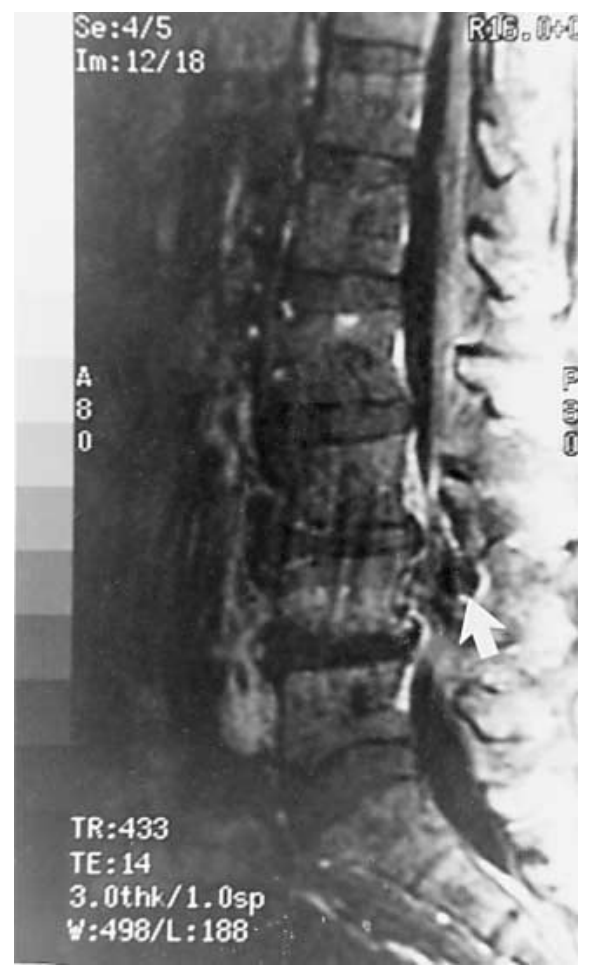

Fig. 3. MRI of the lumbosacral spine with (a) and without (b) gadolinium contrast showing discitis (arrow head) and osteomyelitis (arrow) at the L5-S1 level. Biopsy revealed acute and chronic inflammation with necrosis, and culture grew Staphylococcus aureus.
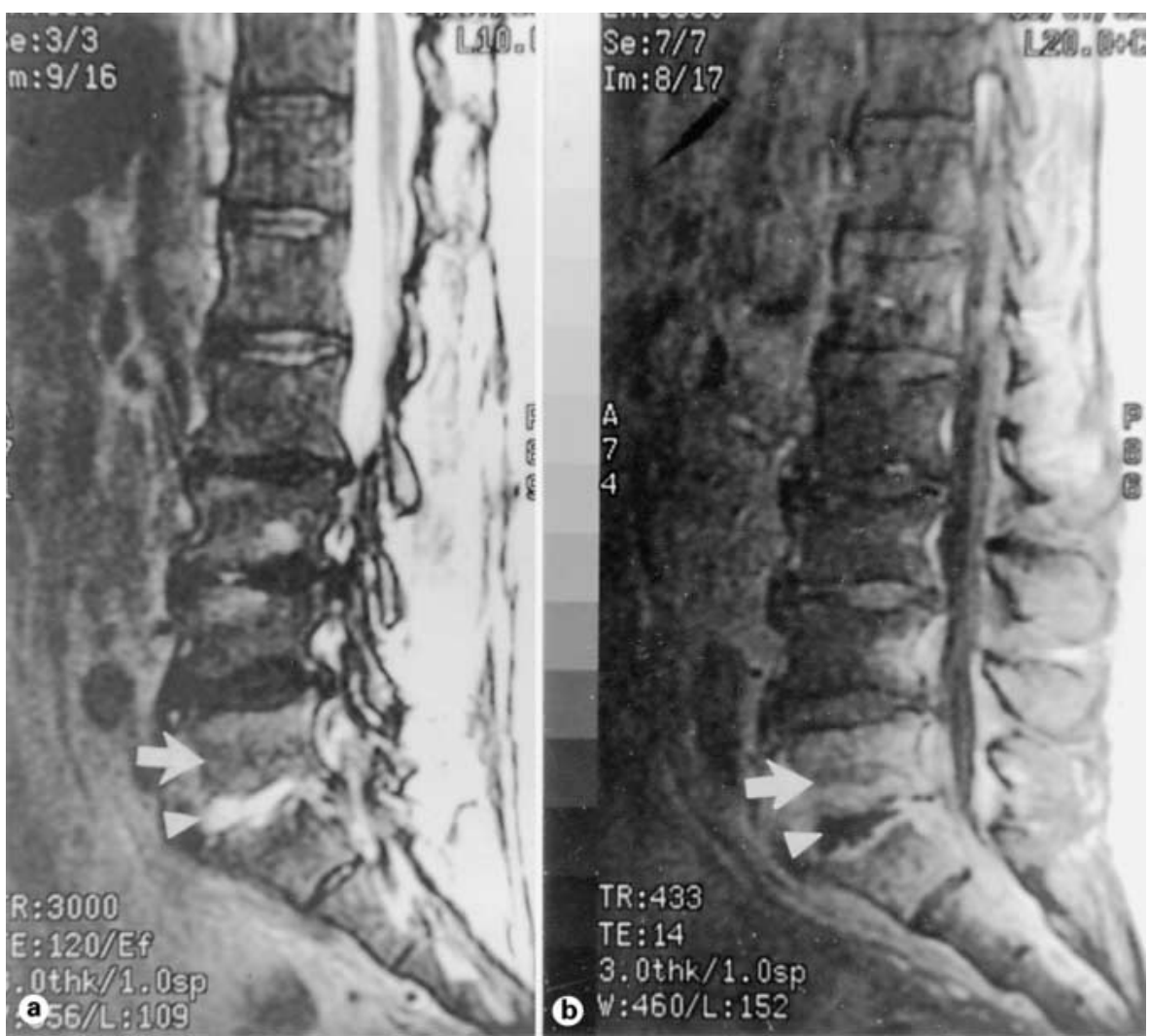

Routine Replacement of HD Catheter

Am J Nephrol 2003;23:202-207 
Fig. 4. MRI of the lumbosacral spine without gadolinium contrast. a A T1-weighted image. b A T2-weighted image. Osteomyelitis (arrow) and discitis (arrow head) involving L2 and L3 are present. Biopsy revealed acute inflammation, and culture grew coagulase-negative Staphylococcus aureus.
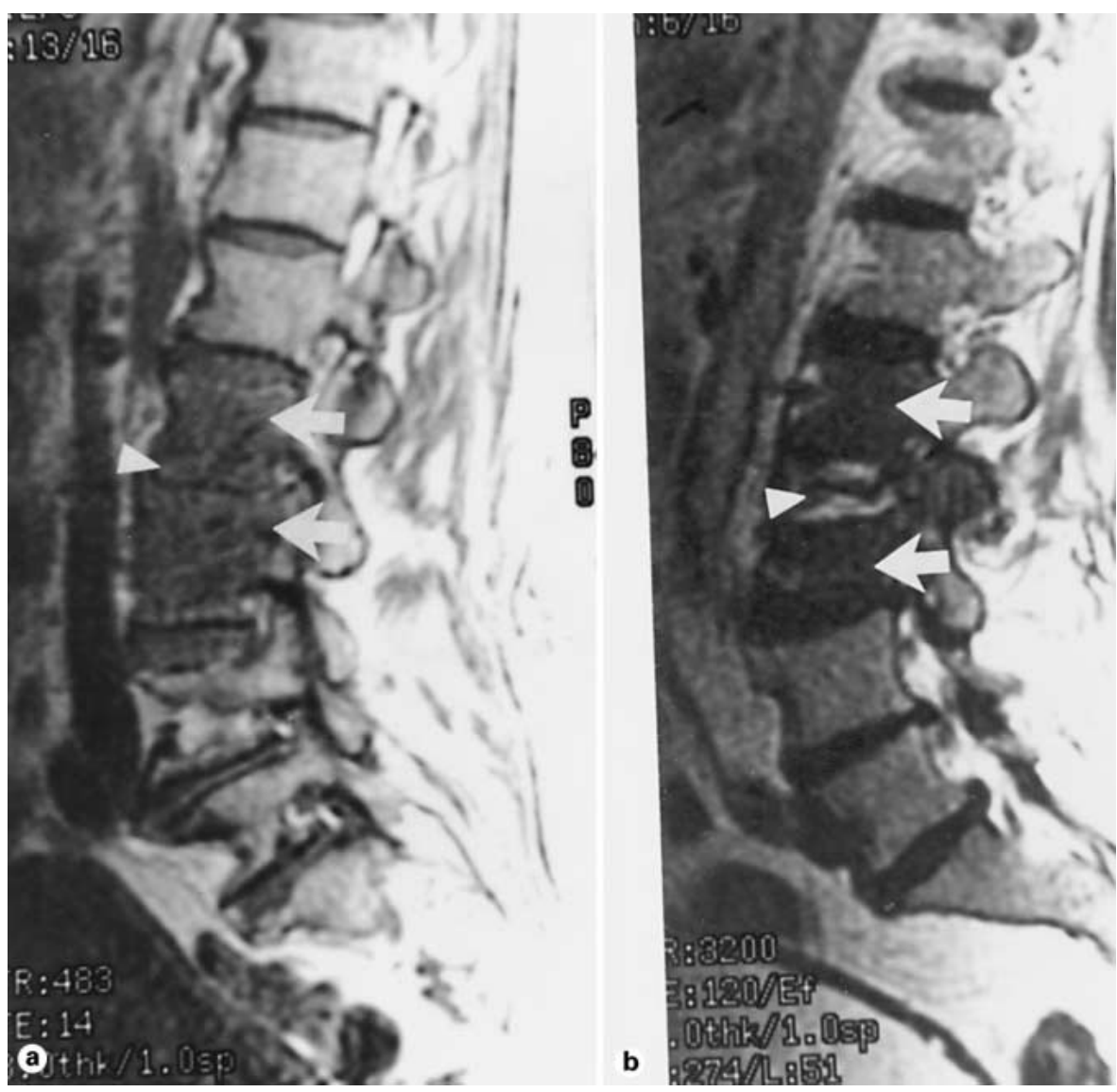

In the former part of the study (1996-1999), systemic antibiotic therapy was continued for $\sim 3$ weeks in an attempt to sterilize the catheter. In the latter part of the study, the systemic antibiotics were discontinued 2-3 weeks after the catheter had been exchanged over a guide wire.

During the 20 months prior to the change in our protocol, we documented 38 bacteremic episodes in our HD population, of which $86.5 \%$ were with gram-positive microorganisms ( $S$. aureus $25 \%$, coagulase-negative staphylococcus $65 \%$, enterococci $10 \%$ ), $11 \%$ with gram-negative microorganisms, and $2.5 \%$ with yeast infection. During the 15 months after the change in our protocol, we documented 34 bacteremic episodes, of which $82 \%$ were with gram-positive microorganisms $(S$. aureus $31.5 \%$, coagulase-negative staphylococcus $52.5 \%$, enterococci $16 \%$ ), $14 \%$ with gram-negative microorganisms, and $4 \%$ with yeast infection. Thus, the incidence of bacteremia and their microbiology were very similar in the two time periods. Moreover, we found a similar duration of hospital- ization for documented bacteremic episodes in each time period (mean $\pm \mathrm{SD}, 5.2 \pm 4.5$ vs. $6.3 \pm 5.7$ days, $\mathrm{p}=0.4$, systemic antibiotics alone vs. catheter exchange after initiation of antibiotic treatment, respectively).

\section{Discussion}

The native AV fistulas, followed by the synthetic AV grafts, are considered optimal long-term vascular accesses for patients on maintenance HD. However, some patients require dual-lumen, tunneled, cuffed HD catheters, either temporarily while awaiting maturation of a native AV fistula or development of a newly placed synthetic AV graft, or as a last resort due to lack of other long-term HD accesses.

Bacteremia is a common complication in HD patients with central HD catheters. These patients are typically treated with a 2- to 3-week course of parenteral antibiotics. Most nephrologists will attempt to salvage the HD 
catheter unless the clinical condition of the patient deteriorates or the bacteremia does not resolve. Although the management of bacteremia might initially seem successful, secondary or metastatic infectious complications such as paraspinal/vertebral abscess and endocarditis remain a concern.

Kovalik et al. [6] reported 10 cases of epidural abscesses in about 400 maintenance HD patients over a 5year period. Eight patients had dual-lumen, tunneled, HD catheters. The epidural abscesses appeared to be related to the previous episodes of bacteremia in most, if not all of the patients. They concluded that attempts at catheter salvage with parenteral antibiotics alone have significant risks for complications, such as epidural abscess formation.

We encountered 8 cases of paraspinal/vertebral infections, all of whom had HD access-associated bacteremia in the preceding 1-20 weeks. Catheter salvage had been attempted in these patients with systemic antibiotic therapy alone. Subsequently, we changed our protocol for the management of bacteremia in patients with tunneled, cuffed, HD catheters, i.e., the catheters were exchanged at $\sim 48 \mathrm{~h}$ over a guide wire using the old tunnel or a new tunnel (if there was evidence of tunnel infection), as has been advocated before [9]. Following this change in our clinical practice, we did not encounter any new cases of paraspinal/vertebral infection(s) at our institution over the next 15-month period. This was in view of roughly the same number of bacteremic episodes (38 episodes over 20 months vs. 34 episodes over 15 months) and their micro- biology ( 86.5 vs. $82 \%$ gram-positive staphylococci; 11 vs. $14 \%$ gram-negative bacilli; 2.5 vs. $4 \%$ yeast infection) in the two time periods. Thus, despite the similar incidence of de novo catheter-associated bacteremia in these two time periods, we noticed metastatic infectious complications in the former period when catheter salvage was routinely attempted with systemic antibiotics alone, potentially leading to more prolonged clinical or subclinical bacteremia. We also found a similar duration of hospitalization for the documented episodes of bacteremia in the two time periods, which suggests that subclinical and/or intermittent bacteremia in the former time period might have been responsible for the high incidence of paraspinal/vertebral infection in them.

In summary, our observation illustrates that HD access-associated bacteremia may result in serious complication of paraspinal/vertebral infection(s). The development of these metastatic infections may occur up to weeks after successful treatment of the original bacteremia. Thus, a new onset of back pain in a bacteremic or recently documented bacteremic HD patient should prompt diagnostic efforts to exclude this serious complication. Replacement or exchange of tunneled, cuffed catheters over a guide wire has helped us eliminate paraspinal/vertebral infections at our institution. Therefore, we strongly discourage the practice of catheter salvage with systemic antibiotics alone and recommend catheter replacement at $\sim 48 \mathrm{~h}$ of systemic antibiotic therapy to prevent this potential infectious complication.

\section{References}

1 Schwab SJ, Buller GL, McCann RL: Prospective evaluation of a Dacron cuffed hemodialysis catheter for prolonged use. Am J Kidney Dis 1988;9:166-169.

2 Kaufman JL, Chang BB, Shah DM, Leather RP: Hemodialysis access catastrophes. Semin Dial 1991;4:33-39.

3 Shusterman NH, Kloss K, Mullen JL: Successful use of double-lumen, silicone rubber catheters for permanent hemodialysis access. Kidney Int 1989;35:887-890.

4 Marr KA, Sexton DJ, Conlon MB: Catheterrelated bacteremia and outcome of attempted catheter salvage in patients undergoing hemodialysis. Ann Intern Med 1997;127:275-280.

5 Moss A, Vasilakis C, Holley J: Use of a silicon dual-lumen catheter with a Dacron cuff as a long-term vascular assess for hemodialysis patients. Am J Kidney Dis 1990;16:211-215.
6 Kovalik EC, Raymond JR, Albers FJ: A clustering of epidural abscesses in chronic hemodialysis patients: Risks of salvaging access catheters in cases of infection. J Am Soc Nephrol 1996; 7:2264-2267.

7 Swartz RD, Messana JM, Boyer CJ: Successful use of cuffed central venous hemodialysis catheters inserted percutaneously. J Am Soc Nephrol 1994:4:1719-1725.

8 National Kidney Foundation: K/DOQI clinical practice guidelines for vascular access, 2000. Am J Kidney Dis 2001;37(suppl 1):S137S181.

9 Saad TF: Bacteremia associated with tunneled, cuffed hemodialysis catheters. Am J Kidney Dis 1999;34:1114-1124.

10 Bastani B, Minton J, Islam, S: Insufficient penetration of systemic vancomycin into the PermCath lumen. Nephrol Dial Transplant 2000;15:1035-1037.
11 Costerton JW, Stewart PS, Greenberg EP: Bacterial biofilms: A common cause of persistent infections. Science 1999;284:1318-1322.

12 Passerini L, Lam K, Costerton JW, King EG: Biofilms on indwelling vascular catheters. Crit Care Med 1992;20:665-673.

13 Capdevila JA, Separra A, Planes AM: Successful treatment of hemodialysis catheter-related sepsis without catheter removal. Nephrol Dial Transplant 1993;8:231-234.

14 Andris DA, Krzywda EA, Edmiston CE, et al: Elimination of intraluminal colonization by antibiotic lock in silicone vascular catheters. Nutrition 1998;14:427-432.

15 Danner RL, Hartman BJ: Update of spinal epidural abscess: 35 cases and review of literature. Rev Infect Dis 1987;9:265-274. 